\title{
The cognitive and neural bases of human tool use
}

\author{
François Osiurak $^{1,2 *}$ and Cristina Massen ${ }^{3,4}$ \\ ${ }^{1}$ Laboratoire d'Etude des Mécanismes Cognitifs, Université Lyon 2, Lyon, France \\ 2 Institut Universitaire de France, Paris, France \\ ${ }^{3}$ Leibniz Research Centre for Working Environment and Human Factors, Dortmund, Germany \\ ${ }^{4}$ Bonn-Rhein-Sieg University of Applied Sciences, Rheinbach, Germany \\ *Correspondence: francois.osiurak@univ-lyon2.fr
}

Edited and reviewed by:

Bernhard Hommel, Leiden University, Netherlands

Keywords: tool use, action, motor control, neural bases, cognitive bases

It is a euphemism to say that humans use tools. Humans possess a vast repertoire of tools they use every day. In fact, as language or bipedal locomotion, tool use is a hallmark of humans. Tool use has also been often viewed as an important step during evolution (van Schaik et al., 1999) or even as a marker of the evolution of human intelligence (Wynn, 1985). So a fundamental issue is, what are the cognitive and neural bases of human tool use? The present series of papers in this special topic represents the newest additions to that research topic.

Central to that topic is the issue of the nature of the representations underlying tool use. Most of our understanding has come from the study of brain-damaged patients with tool use disorders, also called apraxia of tool use. When asked to light a candle, for example, those patients can light the candle correctly but then put it to the mouth in an attempt to smoke it. Such observations have led traditional cognitive models of apraxia to assume that tool use is supported by sensorimotor knowledge about tool manipulation (e.g., Rothi et al., 1991; Buxbaum, 2001). Consistent with this, Gainotti (2013) reviews a series of neuropsychological and neuroimaging studies indicating that perceptual, motor, and encyclopedic sources of knowledge have different weights in the construction of the different object categories (i.e., living things, tools) that are stored within the brain. This sensorymotor hypothesis assumes that manipulation knowledge stored within inferior fronto-parietal areas is critical to tool use skills. This link is also suggested by van Elk (2014), who conducted an fMRI study wherein participants had to predict the subsequent use of a presented tool. His results indicate that the left inferior parietal lobe might store hand-posture representations that can be used for planning tool-directed actions as well as for predicting other's actions.

Contrary to the traditional cognitive models of apraxia, a growing body of literature suggests that the left inferior parietal lobe might rather support technical reasoning, namely, the ability to reason about physical object properties (Goldenberg and Spatt, 2009; Osiurak et al., 2009, 2010, 2013; Goldenberg, 2013; Osiurak, 2014). Support for the technical reasoning hypothesis comes from findings demonstrating a strong association in left brain-damaged patients between the ability to use familiar tools and the ability to use novel tools to solve mechanical problems (for reviews, see Goldenberg, 2013; Osiurak, 2014). Four review articles of this special issue also provide evidence in line with the technical reasoning hypothesis. Bienkiewicz et al. (2014), Orban and Caruana (2014), and Vingerhoets (2014) emphasize that the ability to understand mechanical actions might be the specificity of the anterior portions of the inferior parietal lobe (particularly the supramarginal gyrus) while the posterior parietal cortex might be involved in the planning of the grasping and reaching components of both tool-use and non-tool-use actions. In the same vein, by reviewing studies investigating tool use disorders in left brain-damaged patients over the last 30 years, Baumard et al. (2014) suggest that the loss of mechanical knowledge might be the core deficit in left brain-damaged patients with apraxia of tool use.

Two experimental articles also address the issue of the involvement of mechanical vs. manipulation knowledge in tool use. First, Parry et al. (2014) examine both functional dynamics (i.e., the understanding of the mechanical actions involved in the task) and joint contribution profiles of participants with different levels of expertise in a primordial percussive task (i.e., production of stone flakes using the Oldowan method). Their results show that when people learn a tool use activity what they learn is the functional dynamics rather than any particular movement per se. Second, Müsseler et al. (2014) asked participants to use lever tools or to imagine using them in order to explore the role played in response generation by the spatial compatibility relationships between stimulus ( $S$; at which the effect points of the lever aims at), responding hand (R) and effect point of the lever (E). They observed that the most prominent compatibility effects were for RE compatibility, corroborating the idea that even in tool use planning is influenced not only by the spatial relationship between stimulus and response, but also by the intended action effects. Similar results are reported by Rieger et al. (2014), who had participants perform circling movements with a stylus (movement) and presented distorted visual feedback of the movements on a screen (visual effect). When participants had to synchronize the visual feedback dot with a second, rotating stimulus on the screen (stimulus), strong compatibility effects emerged for the relationship between the hand movement (response) and the visual effect of this movement on the screen.

As Fagard et al. (2014) state, the development of tool use in human infants has received little interest until recently. For example, an unresolved issue is whether tool use appears through sudden insight or emerges progressively through familiarization with experience. Fagard et al. (2014) address this issue by conducting a longitudinal study on five infants from age 12 to 20 
months. Children have to use a rake-like tool to reach toys presented out of reach. Their results indicate that it is only between 16 and 20 months that the infants suddenly start to intentionally try to bring the toy closer with the tool. For them, this sudden success at about 18 months might correspond to the coming together of a variety of capacities, such as the development of means-end behavior.

Tools are also specific because they modify our perception of the world. For instance, it is known that using a tool can alter space perception in that far stimuli become processed as if they were nearer (Maravita and Iriki, 2004; Witt et al., 2005; Osiurak et al., 2012). Likewise, body representations can be modified when using a tool so that the tool is incorporated and becomes part of our body (Iriki et al., 1996; Cardinalli et al., 2009). An interesting issue, however, is whether these modifications only occur after the real use of tools or can also appear in a tool-use imagery condition. Baccarini et al. (2014) provide a positive answer to this issue by showing that tool-use imagery is sufficient to affect the representation of the user's arm.

In line with the view of common representations for perception, imagery, and action, Kelly and Wheaton (2013) investigate the understanding of tool-use actions viewed from different perspectives and conclude that perception and understanding is facilitated when tool-use actions are viewed from an egocentric (as opposed to allocentric) perspective.

Finally, two theoretical papers also contribute to this special topic on broader issues. In line with the extended mind view, Borghi et al. (2013) suggest that words can be conceived as quasiexternal devices (or tools) that extend our cognition. For example, words function like tools because they also enlarge the bodily space of action and, as a result, modify our sense of body. Baber et al. (2014) propose the notion of distributed cognition to account that tool use is not only based on internal representations (e.g., manipulation knowledge or mechanical knowledge) but also external representations such as the location of tools within the workspace.

In sum, this special issue includes a series of articles from neuropsychology, neuroimaging, experimental psychology, developmental psychology, and ergonomics that provide very interesting findings and open new issues for future research on the topic. Let's hope that we possess the good tools to solve them!

\section{ACKNOWLEDGMENTS}

This work was supported by grants from ANR (Agence Nationale pour la Recherche; Project "Dèmences et Utilisation d'Outils/Dementia and Tool Use," NANR 2011 MALZ 006 03; François Osiurak; Project "Cognition et économie liée à l'outil/Cognition and tool-use economy" ECOTOOL, François Osiurak), and was performed within the framework of the LABEX CORTEX (ANR-11-LABX-0042) of Universitè de Lyon (François Osiurak), within the program "Investissements d'Avenir" (ANR11- IDEX-0007) operated by the French National Research Agency (ANR).

\section{REFERENCES}

Baber, C., Parekh, M., and Cengiz, T. G. (2014). Tool use as distributed cognition: how tools help, hinder and define manual skill. Front. Psychol. 5:116. doi: 10.3389/fpsyg.2014.00116
Baccarini, M., Martel, M., Cardinalli, L., Sillan, O., Farnè, A., and Roy, A. C. (2014). Tool use imagery triggers tool incorporation in the body schema. Front. Psychol. 5:492. doi: 10.3389/fpsyg.2014.00492

Baumard, J., Osiurak, F., Lesourd, M., and Le Gall, D. (2014). Tool use disorders after left brain damage. Front. Psychol. 5:473. doi: 10.3389/fpsyg.2014.00473

Bienkiewicz, M. M. N., Brandi, M.-L., Goldenberg, G., Hughes, C. M. L., and Hermsdörfer, J. (2014). The tool in the brain: apraxia in ADL. Behavioral and neurological correlates of apraxia in daily living. Front. Psychol. 5:353. doi: 10.3389/fpsyg.2014.00353

Borghi, A. M., Scorolli, C., Caligiore, D., Baldassare, G., and Tummolini, L. (2013). The embodied mind extended: using words as social tools. Front. Psychol. 4:214. doi: 10.3389/fpsyg.2013.00214

Buxbaum, L. J. (2001). Ideomotor apraxia: a call to action. Neurocase 7, 445-448. doi: 10.1093/neucas/7.6.445

Cardinalli, L., Frassinetti, F., Brozzoli, C., Urquizar, C., Roy, A. C., and Farnè, A. (2009). Tool-use induces morphological updating of the body schema. Curr. Biol. 19, 478-479. doi: 10.1016/j.cub.2009.05.009

Fagard, J., Rat-Fischer, L., and O'Reagan, J. K. (2014). The emergence of use of a rake-like tool: a longitudinal study in human infants. Front. Psychol. 5:491. doi: 10.3389/fpsyg.2014.00491

Gainotti, G. (2013). Controversies over the mechanisms underlying the crucial role of the left fronto-parietal areas in the representation of tools. Front. Psychol. 4:727. doi: 10.3389/fpsyg.2013.00727

Goldenberg, G. (2013). Apraxia: The Cognitive Side of Motor Control. Oxford: Oxford University Press. doi: 10.1093/acprof:oso/9780199591510.001.0001

Goldenberg, G., and Spatt, J. (2009). The neural basis of tool use. Brain 132, 1645-1655. doi: 10.1093/brain/awp080

Iriki, A., Tanaka, M., and Iwamura, Y. (1996). Coding of modified body schema during tool use by macaque postcentral neurones. Neuroreport 7, 2325-2330. doi: 10.1097/00001756-199610020-00010

Kelly, R. L., and Wheaton, L. A. (2013). Differential mechanisms of action understanding in left and right-handed subjects: the role of perspective and handedness. Front. Psychol. 4:957. doi: 10.3389/fpsyg.2013.00957

Maravita, A., and Iriki, A. (2004). Tools for the body (schema). Trends Cogn. Sci. 8, 79-86. doi: 10.1016/j.tics.2003.12.008

Müsseler, J., Wühr, P., and Ziessler, M. (2014). Using tools with real and imagined tool movements. Front. Psychol. 5:515. doi: 10.3389/fpsyg.2014.00515

Orban, G. A., and Caruana, F. (2014). The neural basis of human tool use. Front. Psychol. 5:310. doi: 10.3389/fpsyg.2014.00310

Osiurak, F. (2014). What neuropsychology tells us about human tool use? The four constraints theory (4CT): mechanics, space, time and effort. Neuropsychol. Rev. 24, 88-115. doi: 10.1007/s11065-014-9260-y

Osiurak, F., Jarry, C., Allain, P., Aubin, G., Etcharry-Bouyx, F., Richard, I., et al. (2009). Unusual use of objects after unilateral brain damage. The technical reasoning model.Cortex 45, 769-783. doi: 10.1016/j.cortex.2008.06.013

Osiurak, F., Jarry, C., and Le Gall, D. (2010). Grasping the affordances, understanding the reasoning. Toward a dialectical theory of human tool use. Psychol. Rev. 117, 517-540. doi: 10.1037/a0019004

Osiurak, F., Jarry, C., Lesourd, M., Baumard, J., and Le Gall, D. (2013). Mechanical problem-solving in left brain-damaged patients. Neuropsychologia 51, 1964-1972. doi: 10.1016/j.neuropsychologia.2013.06.017

Osiurak, F., Morgado, N., and Palluel-Germain, R. (2012). Tool use and perceived distance. When unreachable becomes sponatenously reachable. Exp. Brain Res. 218, 331-339. doi: 10.1007/s00221-012-3036-5

Parry, R., Dietrich, G., and Bril, B. (2014). Tool use ability depends on understanding of functional dynamics and not specific joint contribution profiles. Front. Psychol. 5:306. doi: 10.3389/fpsyg.2014.00306

Rieger, M., Dietrich, S., and Prinz, W. (2014). Effects of angular shift transformations between movements and their visual feedback on coordination in unimanual circling. Front. Psychol. 5:693. doi: 10.3389/fpsyg.2014.00693

Rothi, L. J. G., Ochipa, C., and Heilman, K. M. (1991). A cognitive neuropsychological model of limb praxis. Cogn. Neuropsychol. 8, 443-458. doi: 10.1080/02643299108253382

van Elk, M. (2014). The left inferior parietal lobe represents stored hand postures for object use and action prediction. Front. Psychol. 5:333. doi: 10.3389/fpsyg.2014.00333

van Schaik, C. P., Deaner, R. O., and Merrill, M. Y. (1999). The conditions for too use in primates: Implications for the evolution of material culture. J. Hum. Evol. 36, 19-41. doi: 10.1006/jhev.1999.0304 
Vingerhoets, G. (2014). Contribution of the posterior parietal cortex in reaching, grasping, and using objects and tools. Front. Psychol. 5:151. doi: 10.3389/fpsyg.2014.00151

Witt, J. K., Proffitt, D. R., and Epstein, W. (2005). Tool use affects perceived distance, but only when you intend to use it. J. Exp. Psychol. Hum. Percept. Perform. 31, 880-888. doi: 10.1037/0096-1523.31.5.880

Wynn, T. (1985). Piaget, stone tools and the evolution of human intelligence. World Archaeol. 17, 32-43. doi: 10.1080/00438243.1985.9979948

Conflict of Interest Statement: The authors declare that the research was conducted in the absence of any commercial or financial relationships that could be construed as a potential conflict of interest.
Received: 11 September 2014; accepted: 12 September 2014; published online: 06 October 2014.

Citation: Osiurak F and Massen C (2014) The cognitive and neural bases of human tool use. Front. Psychol. 5:1107. doi: 10.3389/fpsyg.2014.01107

This article was submitted to Cognition, a section of the journal Frontiers in Psychology.

Copyright (C) 2014 Osiurak and Massen. This is an open-access article distributed under the terms of the Creative Commons Attribution License (CC BY). The use, distribution or reproduction in other forums is permitted, provided the original author(s) or licensor are credited and that the original publication in this journal is cited, in accordance with accepted academic practice. No use, distribution or reproduction is permitted which does not comply with these terms. 